\title{
Renal consequences of lymphoproliferative disorders and monoclonal gammopathy
}

\begin{abstract}
Background: Kidney damage in lymphoproliferative disorders (LPD) include specific infiltration, different types of paraprotein deposition, paraneoplastic glomerulonephritis (GN), treatment complications and others. Paraproteinemia in absence of overt LPD, formerly known as monoclonal gammopathy of undetermined significance (MGUS), now is considered to be monoclonal gammopathy of renal significance (MGRS) in many cases. We aimed to evaluate incidence of different types of kidney lesions in patients, admitted to nephrology unit and diagnosed with LPD/MGRS
\end{abstract}

Methods: Using electronic database for 1994-1014 we searched 282 patients with LPD/ MGRS- $2.8 \%$ out of 9916 of primary admissions. Work-up included serum and urine immune electrophoresis, bone marrow aspiration and/or biopsy and/or lymph node biopsy with light microscopy and immune histochemistry, peripheral blood immune phenol typing, skeletal X-ray, chest and abdomen CT and kidney biopsy with light microscopy and immune histology, and occasionally electron microscopy. Cases not verified by kidney pathology were excluded from further analysis.

Results: Study group comprised of 139 patients, 77 (55.4\%) male and 62 (44.6\%) female, median age $64.5[17 ; 81]$ years, with 146 pathology patterns-in 6 patients 2 or 3 variants of damage were found simultaneously or consequently. Amyloidosis presented in 96 cases (92- AL, 1-AH and $3 \mathrm{AA}$ amyloidosis); light/heavy chain deposition disease and specific infiltration-in 9 cases each; cast-nephropathy-in 6; membranous nephropathy-in 5; nephrosclerosis-in 4; proliferative $\mathrm{GN}$ with monoclonal $\mathrm{IgG} / \mathrm{IgA}$ deposits, focal segmental glomerulosclerosis and tubulointerstitial nephritis-in 3 cases each; Cryoglobulinemic GN, GN with monoclonal IgM deposits, crescentic GN and minimal changes-in 2 cases each. Paraproteinemic, mostly organized lesions dominated, with $\mathrm{AL}$ amyloidosis constituting $63 \%$ of cases, mainly as "primary" amyloidosis.

Conclusion: Spectrum of kidney damage in our cohort included 16 variants. LPD and MGRS share common pathology features, kidney biopsy is mandatory to confirm diagnosis and establish the link between LPD/MGRS and kidney damage.

Keywords: amyloidosis, kidney biopsy, lymphoproliferative disorders, monoclonal gammopathy, multiple myeloma, nephropathy
Volume 2 Issue 4 - 2015

\author{
Elena V Zakharova, ${ }^{1,2}$ Ekaterina S \\ Stolyarevich ${ }^{2,3}$ \\ 'Department of Nephrology, Moscow City Clinical Hospital n.a. \\ S.P. Botkin, Russia \\ ${ }^{2}$ Chair of Nephrology, Moscow State University of Medicine and \\ Dentistry, Russia \\ ${ }^{3}$ Department of Pathology, Moscow City Nephrology Center, \\ Russia
}

Correspondence: Elena $\vee$ Zakharova, State University of Medicine and Dentistry, City Clinical Hospital n.a. S.P. Botkin, I I5284, 2-ndBotkinskyproezd, 5, Moscow, Russia, Tel +7 499728 829I,+7 495945 1756, Email helena.zakharova@gmail.com

Received: May 04, 2015 | Published: July 30, 2015
Abbreviations: AA, AA amyloidosis; AH, ah amyloidosis; AKI, acute kidney injury; AL, al amyloidosis; $C D$, castleman's disease; CGN, crescentic glomerulonephritis; CKD, chronic kidney disease; Cryo GN, cryoglobulinemic glomerulonephritis; CN, cast-nephropathy; DNS, diffuse nephrosclerosis; FD, franklin's disease; FSGS, focal segmental glomerulosclerosis; GN, glomerulonephritis; GOMMID, glomerulonephritis with organized microtubular monoclonal deposits; HCD, heavy chain diseases; HCDD, heavy chain deposition disease; HD, hodgkin's disease; IgA, immunoglobulin a; IgG, immumoglobulin g; IgM, immunoglobulin m; LCDD, light chain deposition disease; LPD, lymphoproliferative disorders; MGRS, monoclonal gammopathy of renal significance; MGUS, monoclonal gammopathy of undetermined significance; MCD, minimal change disease; MIDD monoclonal immunoglobulin deposition disease; $\mathrm{MN}$, membranous nephropathy; NHL/CLL, non-hodgkin lymphoma/leukemia; NS, nephrotic syndrome; PGNMID, proliferative GN with monoclonal immunoglobulin deposits; SI, specific infiltration; TIN, tubulointerstitial nephritis; WM, waldenström's macroglobulinemia

\section{Introduction}

LPD represent a huge and hetero generous group of diseases with a broad spectrum of clinical presentation, including variety of kidney lesions. Importantly, symptoms of kidney damage may dominate and even preclude vert LPD's. Renal involvement is defined by numerous mechanisms: tumor mass localization; clonal cell expansion; deposition of secreted paraproteins, including organized (crystals, fibrils, microtubules), and non-organized deposits of monoclonal immunoglobulin's or fragments thereof; hormones, cytokines and growth factors secretion; metabolic, electrolyte and coagulation disturbances; infection and drug-induced complications etc. (Table 1), affecting urinary tract, renal arteries and veins and all parenchymal compartments-intra-renal vasculature, glomeruli, tubules and interstitial space, sometimes simultaneously. ${ }^{1-12}$

Patients may present with acute kidney injury (AKI), nephrotic syndrome (NS), proteinuria and/or hematuria, arterial hypertension or chronic kidney disease (CKD). Differential diagnostics is the major challenge, often demanding pathology evaluation. Beyond well 
known cast-nephropathy $(\mathrm{CN})$ and $\mathrm{AL}$ amyloidosis $(\mathrm{AL})$, patients with Multiple Myeloma (MM), Waldenström's Macroglobulinemia (WM), Heavy Chain Diseases(HCD), Non-Hodgkin Lymphoma/Leukemia (NHL/CLL), Hodgkin's disease (HD) and Castleman's Disease (CD) may present with monoclonal immunoglobulin deposition disease (MIDD)-light chain/heavy chain deposition disease (LCDD/HCDD), proliferative glomerulonephritis with monoclonal deposits of IgG and IgA (PGNMID), glomerulonephritis with organized microtubular monoclonal deposits (GOMMID), Cryoglobulinemic GN (Cryo GN) and paraneoplastic GN's, AA amyloidosis (AA), light-chain tubulopathy etc. see Table 1 , which cannot be differentiated solely on the base of clinical features. ${ }^{1,5,7,9,11,12}$

Table I Mechanisms of kidney damage in lympho proliferative disorders

\begin{tabular}{l}
\hline Paraprotein deposition \\
\hline Organized paraprotein deposits \\
\\
- $\quad$ Cast-nephropathy \\
- $\quad$ Immuired Fanconi's syndrome (light chain tubulopathy) \\
Cryoglobulinemic glomerulonephritis \\
monoclonal deposits (Immunotactoid Glomerulonephritis)
\end{tabular}

\section{Without paraprotein deposition}

\section{Specific lymphoid and plasma cell infiltration}

Paraneoplastic glomerulonephritis

$\bullet$

- Membranous nephropathy

- Focal segmental glomerulosclerosis

- Crescentic glomerulonephritis

\section{Hyperviscosity syndrome}

Metabolic nephropathies

- $\quad$ Tumor-lysis syndrome (uric acid/phosphate nephropathy)

- Hypercalcemia-induced acute kidney injury

Pre-renal acute kidney injury
Non-organized paraprotein deposition

Monoclonal immunoglobulin deposition disease
- $\quad$ Proliferative glomerulonephritis with monoclonal
immunoglobulin deposits (Nasr disease)
Glomerulonephritis with monoclonal intra capillary
IgM deposits

Thrombotic damages

- $\quad$ Renal vein thrombosis

- Thrombotic micro angiopathy

\section{Urinary tract obstruction}

- $\quad$ Lymph nodes compression

- Retroperitoneal fibrosis

Sepsis, Urinary Tract Infections

Radiation-Induced Nephritis

Drug-Induced Nephritis

- Minimal change disease

- Membranous nephropathy

- Interstitial nephritis
Even more challenging are cases with monoclonal gammopathy of undetermined significance (MGUS) and kidney damage other than so-called "primary" AL and "primary" LCDD/HCDD, not least because $80 \%$ of patients with MGUS are known to progress to MM, WM and AL amyloidosis over time. The concept of "dangerous small B cell" clones and more recently introduced concept of monoclonal gammopathy of renal significance (MGRS) ${ }^{13}$ provide the clue to diagnostics and management of such patients. Given the prognosis for LPD's as such and LPD's with kidney involvement in particular is serious, and overall and kidney survival to a considerable extent depend on early diagnostics and target therapy, we used this holistic approach $^{14-16}$ to evaluate incidence of different types of kidney lesions in patients, admitted to nephrology unit and diagnosed with LPD/ MGRS.

\section{Materials and methods}

\section{Patient's population}

In this retrospective study from a single center, using electronic database and purposely designed chart we searched and analyzed data for 282 patients, diagnosed with LPD/MGRS, totaling 2.8\% out of 9916 primary admissions over last 20years (1994-2014).

\section{Work-up}

Work-up, beyond routine, included serum and urine immune electrophoresis; peripheral blood immune phenol typing; abdomen, kidneys and peripheral lymph nodes ultrasound; skeletal X-ray; chest and abdomen CT; bone marrow aspiration and/or biopsy; and/or lymph node biopsy with light microscopy and immune histochemistry. 


\section{Main diagnosis}

Diagnosis of LPD was determined in collaboration with hematologist according to WHO classification: $49.6 \%$ of patients were diagnosed with MM; $10.9 \%$ with NHL/CLL; 3.1\% with WM; $2.8 \%$ with HD and evenly $0.3 \%$ with Franklin's disease (FD) and CD. $33 \%$ of patients did not meet criteria for LPD, and were diagnosed with MGRS: "primary" AL-25.1\%, "primary" LCDD/HCDD-1.7\% or other MGRS- $5.6 \%$ (Figure 1). Totally $74 \%$ of patients, and $89 \%$ out of patients with MM, had not been diagnosed with LPD before admission to nephrology unit (Figure 2).

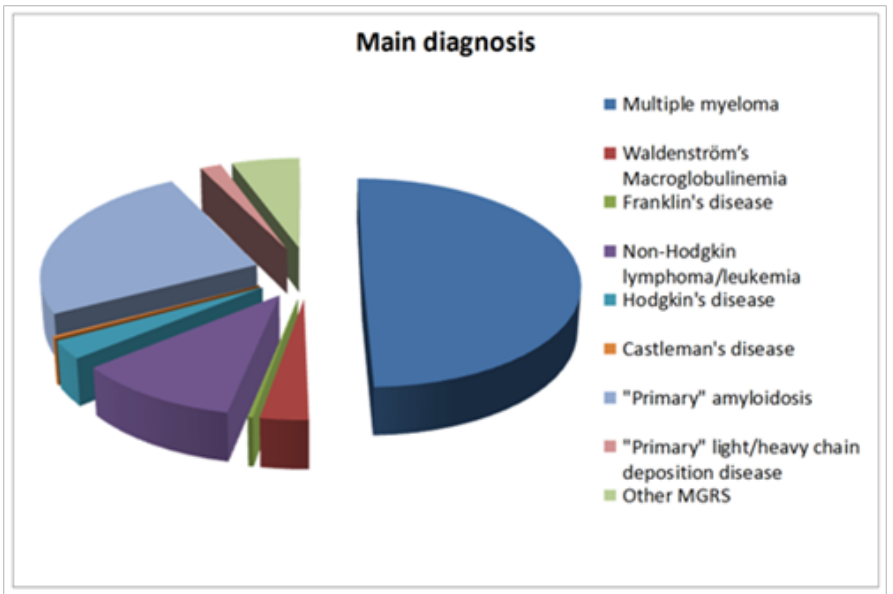

Figure I Main diagnosis in all patients with lymphoproliferative disorders and monoclonal gammopathy of renal significance.

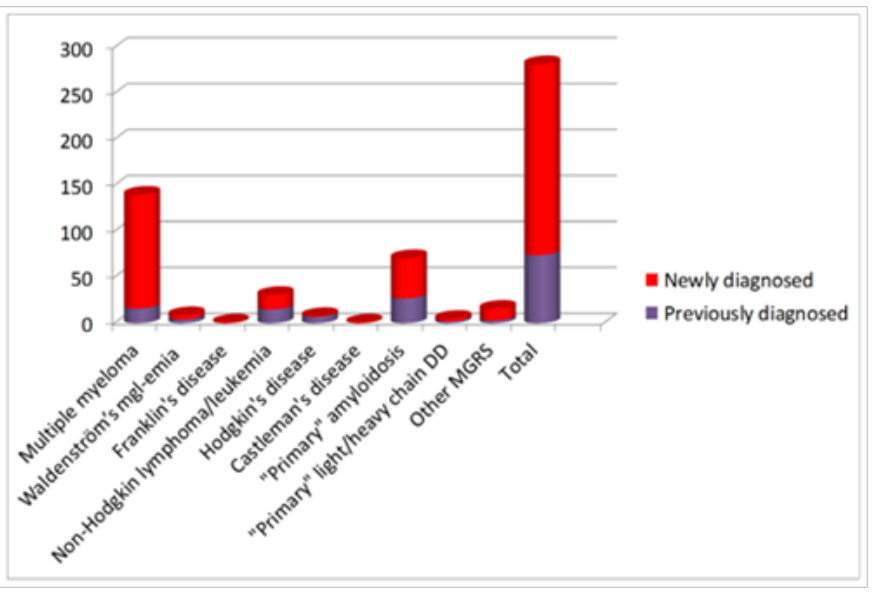

Figure 2 Distribution of newly diagnosed and previously diagnosed cases among all patients with lymphoproliferative disorders and monoclonal gammopathy of renal significance.

\section{Kidney biopsy}

Indications for kidney biopsy included NS, proteinuria $(>0.3 \mathrm{~g} / 24 \mathrm{~h})$ and/or hematuria and/or decreased kidney function (after exclusion of overt MM with AKI, demanding urgent dialysis, intra-and extrarenal tumor masses, urinary tract obstruction, coagulation, electrolyte and metabolic disturbances and infectious complications). Cases not verified by kidney pathology were excluded from further analysis. Kidney core biopsy was taken with BARD-Magnum biopsy guidance facility and evaluated by one pathologist. In selected cases second opinion and/or re-processing and re-evaluation was requested from external pathologists.
Obtained specimens were divided into two or three parts and processed for light microscopy, immune histology, and (in selected cases) electron microscopy. Formalin fixed/paraffin embedded sections for light microscopy was stained with hematoxylin and eosin, Masson's trichrome, periodic acid-Schiff and Congo red. Unfixed cryo-sections were routinely stained for $\operatorname{IgA}$, IgG, IgM, C3, $\mathrm{C} 1 \mathrm{q}$, kappa and lamda light chains and fibrinogen. In selected cases immune fluorescence on formalin fixed/paraffin embedded sections with FITC-conjugated anti IgA, IgG, IgM, C1q, C3, fibrinogen, $\lambda$ and $\kappa$ light chains antibodies, and immune peroxidase staining for amyloid A-protein was performed. Also in selected cases Toluidine blue stained semi-thick sections were examined and 1 glomerulus was identified for electron microscopic study (by external pathologist).

\section{Results and discussion}

Study group comprised $49.2 \%$ of general cohort and included 139 patients, $77(55.4 \%)$ male and $62(44.6 \%)$ female, median age $64.5[17 ; 81$ ]years, with 146 pathology patterns - in 6 patients 2 or 3 variants of damage were found simultaneously or consequently.

\section{Clinical diagnosis and presentation}

Main diagnosis in the study group (Figure 3) turned to be MM in $33(23.7 \%)$ of cases, NHL/CLL-in 14 (10\%), WM-in 6 (4.3\%), HD 5-in (3.6\%), FD and CD-1 (0.7\%) case of each. Totally $79(56.8 \%)$ of patients did not meet LPD diagnostic criteria and were diagnosed with "primary" AL-64 (46\%) cases, "primary" LCDD/HCDD- 5 (3.6\%) cases, and other MGRS-10 (7.2\%) cases. Importantly, that the proportion of MM patients, constituted almost half of general cohort, diminished in the study group to less than quarter of cases $(49.6 \%$ vs $23.7 \%, \mathrm{p}<0.05$ ), reflecting the impact of clinical presentation, including AKI, for the diagnostics of overt MM. And vice versa, proportion of "non-malignant" conditions, such as "primary" AL, "primary" LCDD/HCDD and other MGRS in the study group was much higher, than in the general cohort-56.8\% vs $33 \%(p<0.05)$, which shows the crucial role of pathology for diagnostics of these conditions.

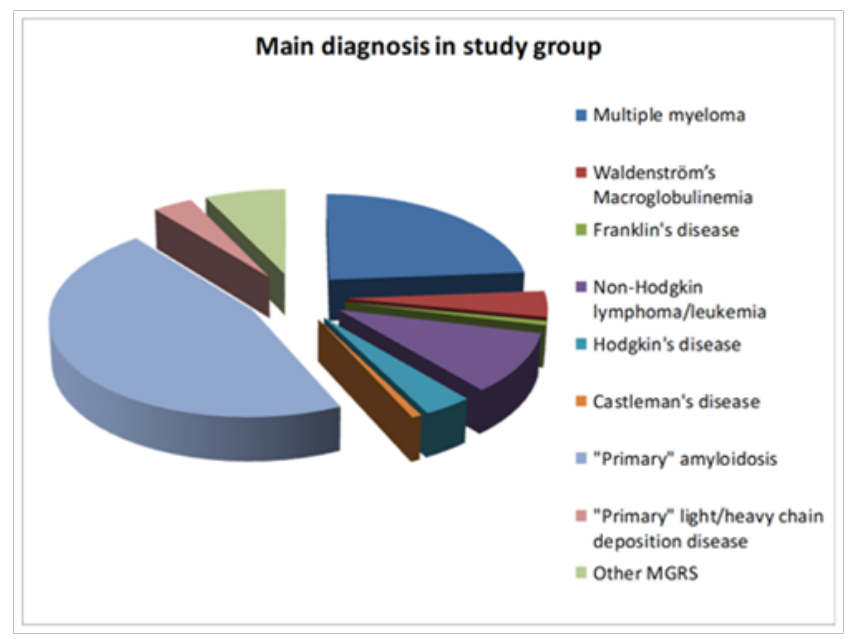

Figure 3 Main diagnosis in the study group with biopsy-proven kidney damage.

$96(69 \%)$ patients presented with NS and $84(60.4 \%)$ had impaired kidney function at the time of biopsy, with $49(35.2 \%)$ presented with both NS and renal dysfunction, and 19 (13.6\%) requiring dialysis at presentation (Figure 4). NS dominated in "primary" AL sub-group (55 out of 64 patients), renal dysfunction was the main clinical feature 
in NHL/CLL (10 out of 14) and MGRS (9 out of 10) sub-groups. Combination of NS and impaired kidney function was seen mainly in WM (4 out of 6), MM (12 out of 33) and "primary" AL (26 out of 64) patients. Dialysis was needed mostly in MM patients (7 out of 33). These peculiarities reflect pathology findings, such as predominantly glomerular vs interstitial involvement in different clinical setting.

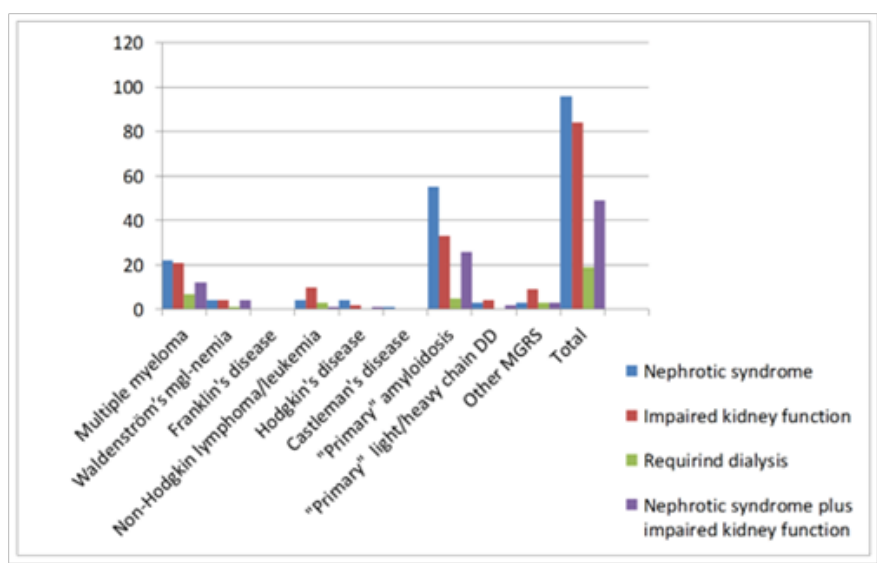

Figure 4 Clinical presentation in the study group with biopsy-proven kidney damage.

\section{Pathology findings}

Pathology data Table 2 showed that kidney damage in LPD/ MGRS patients was associated with variety of lesions, presenting vast majority of patterns, described in the literature. ${ }^{4,5,8,9}$

\section{Paraproteinemic lesions}

Paraprotein deposits comprised totally $78.7 \%$ of pathology findings, mainly represented by AL amyloidosis (63\%), and ten times less frequently-by LCDD/HCDD (6.1\%). CN was found in $4.1 \%$; PGNMID, GN with monoclonal IgM deposits, Cryo GN and AH were also seen, but only in 2-0.6\% respectively. That illustrates that kidney biopsy, performed in every case of NS in adults, may reveal renal amyloidosis and even other types of immunoglobulin deposition more often, than one would expect. On the other hand, relatively small proportion of biopsy-proven $\mathrm{CN}$ is explained by the fact, that some patients with overt MM, presenting with $\mathrm{AKI}$, may not require biopsy and are diagnosed basing on clinical presentation.

\section{Other lesions}

Non-paraproteinemic lesions were presented by different types of $\mathrm{GN}$, such as membranous nephropathy (MN), focal segmental glomerulosclerosis (FSGS), crescentic glomerulonephritis (CGN) and minimal change disease (MCD), totally $8.2 \%$, specific infiltration (SI)-6.1\%, diffuse nephrosclerosis (DNS)-2.7\%, tubulointerstitial nephritis (TIN) and AA amyloidosis- $2 \%$ each, showing the impact of kidney biopsy for differential diagnostics of NS and AKI.

\section{Clinical and pathology correlations}

As it is shown in the Table 3, same pathology findings were often seen in different clinical setting and vice versa.

\section{AL and AA amyloidosis clinical correlations}

AL amyloidosis was not only found as "primary" disease, but also in many cases of $\mathrm{MM}$, in single cases of WM and $\mathrm{CD}$, and also in combination with "primary" LCDD.AA amyloidosis complicated not only HD, which is well known, ${ }^{6}$ but also in a patient with Burkitt lymphoma with spinal cord damage and bed sores.

\section{Cryoglobulinemic glomerulonephritis clinical correlations}

Same way Cryo GN was seen in WM patient and in a case with low grade paraproteinemia without proven LPD, considered as MGRS. $\mathrm{LCDD} / \mathrm{HCDD}$ was found half-to-half in MM and as "primary" in patients without overt LPD.

Table 2 Kidney pathology, representing 139 patients and I 46 variants of lesions

\begin{tabular}{|c|c|c|c|c|c|c|c|}
\hline \multicolumn{4}{|c|}{ Paraprotein deposition I I5 (78.7\%) } & \multicolumn{4}{|c|}{ Without paraprotein deposition 3 I (2I.2\%) } \\
\hline Organized & 101 & Non-organized & 14 & Glomerulonephritis & 12 & Other & 19 \\
\hline $\mathrm{AL}$ amyloidosis & 92 & Light chain deposition disease & 8 & Membranous nephropathy & 5 & Specific infiltration & 9 \\
\hline $\mathrm{AH}$ amyloidosis & I & Heavy chain deposition disease & I & $\begin{array}{l}\text { Crescentic } \\
\text { glomerulonephritis }\end{array}$ & 2 & $\begin{array}{l}\text { Tubulointerstitial } \\
\text { nephritis }\end{array}$ & 3 \\
\hline Cast-nephropathy & 6 & $\begin{array}{l}\text { Glomerulonephritis with monoclonal IgM } \\
\text { deposits }\end{array}$ & 2 & $\begin{array}{l}\text { Focal segmental } \\
\text { glomerulosclerosis }\end{array}$ & 3 & $\begin{array}{l}\text { Diffuse } \\
\text { nephrosclerosis }\end{array}$ & 4 \\
\hline $\begin{array}{l}\text { Cryoglobulinemic } \\
\text { glomerulonephritis }\end{array}$ & 2 & $\begin{array}{l}\text { Proliferative glomerulonephritis with } \\
\text { monoclonal immunoglobulin deposits }\end{array}$ & 3 & Minimal change disease & 2 & AA amyloidosis & 3 \\
\hline
\end{tabular}

\section{Proliferative glomerulonephritis with monoclonal immunoglobulin deposits clinical correlations}

PGNMID was diagnosed in one patient with NHL/CLL, but in the other two cases with low-level monoclonal gammopathy clinical findings and results of work-up were not consistent with any lympho-or plasma cell proliferative disorder, like in original Nasr's description. ${ }^{12}$

\section{Membranous nephropathy and focal segmental glomerulosclerosis clinical correlations}

$\mathrm{MN}$ in paraneoplastic setting was seen in 2 cases of NHL/CLL (in
1 of them in combination with SI of kidneys), and 1 case of WM (we suspect this particular one to be Immunotactoid GN, but were unable to prove this by electron microscopy). 2 other cases, probably also Immunotactoid, were diagnosed as MGRS. FSGS was found in WM, $\mathrm{HD}$ and in 1 case classified as MGRS.

\section{Specific infiltration, tubulointerstitial nephritis and diffuse nephrosclerosis clinical correlations}

Even specific infiltration was not only lymphoid (in one patient combined with MN), but also plasma cell in one case. TIN and DNS were revealed in MM, NHL/CLL and MGRS in more or less equal proportion. 
Table 3 Clinical and pathology correlations (I39 patients, I 46 patterns)

\begin{tabular}{|c|c|c|c|c|c|c|c|c|c|c|}
\hline \multicolumn{2}{|c|}{ PathologylMain diagnosis } & \multirow{2}{*}{$\begin{array}{l}\text { MM } \\
25\end{array}$} & \multirow{2}{*}{$\begin{array}{l}\text { WM } \\
\text { I }\end{array}$} & \multirow{2}{*}{$\begin{array}{l}\text { FD } \\
-\end{array}$} & \multirow{2}{*}{$\begin{array}{l}\text { NHL/ } \\
\text { CLL } \\
-\end{array}$} & \multirow{2}{*}{$\begin{array}{l}\text { HD } \\
-\end{array}$} & \multirow{2}{*}{$\begin{array}{l}\text { CD } \\
\end{array}$} & \multirow{2}{*}{$\begin{array}{l}\text { “Primary” } \\
\text { AL } \\
64\end{array}$} & \multirow{2}{*}{$\begin{array}{l}\text { “Primary” LCDD/ } \\
\text { HCDD } \\
\mathrm{I}\end{array}$} & \multirow{2}{*}{$\begin{array}{l}\text { Other } \\
\text { MGRS } \\
-\end{array}$} \\
\hline $\mathrm{AL}$ & 92 & & & & & & & & & \\
\hline $\mathrm{CN}$ & 6 & 6 & - & - & - & - & - & - & - & - \\
\hline Cryo GN & 2 & - & I & - & - & - & - & - & - & I \\
\hline $\begin{array}{l}\text { GN with lg M } \\
\text { deposits }\end{array}$ & 2 & - & 2 & - & - & - & - & - & - & - \\
\hline PGNMID & 3 & - & - & - & I & - & - & - & - & 2 \\
\hline $\mathrm{MN}$ & 5 & - & 1 & - & 2 & - & - & - & - & 2 \\
\hline CGN & 2 & - & - & - & - & - & - & - & - & 2 \\
\hline MCD & 2 & & - & - & - & 2 & - & - & - & - \\
\hline SI & 9 & I & - & - & 8 & - & - & - & - & - \\
\hline TIN & 3 & - & - & - & 2 & - & - & - & - & I \\
\hline DNS & 4 & I & - & - & 2 & - & - & - & - & I \\
\hline $\mathrm{AA}$ & 3 & - & - & - & I & 2 & - & - & - & - \\
\hline
\end{tabular}

\section{Multiple myeloma and Waldenström's Macroglobulinemia pathology correlations}

MM patients had different pathology patterns: mainly $\mathrm{AL}$ amyloidosis (Figure 5) and CN (Figure 6), but also LCDD/HCDD (Figure 7), SI and DNS. Interestingly, we had a case of MM with three types of lesions-CN, LCDD and AL, showed by two consecutive pathology studies. WM patients were found to have AL, Cryo GN (Figure 8), GN with monoclonal IgM deposits (Figure 9), MN and FSGS.

\section{Non-Hodgkin lymphoma/leukemia and Hodgkin's disease pathology correlations}

NHL/CLL cases were complicated by PGNMID, MN, SI (Figure 10), TIN, DNS and AA amyloidosis; HD-by MCD, FSGS and AA.

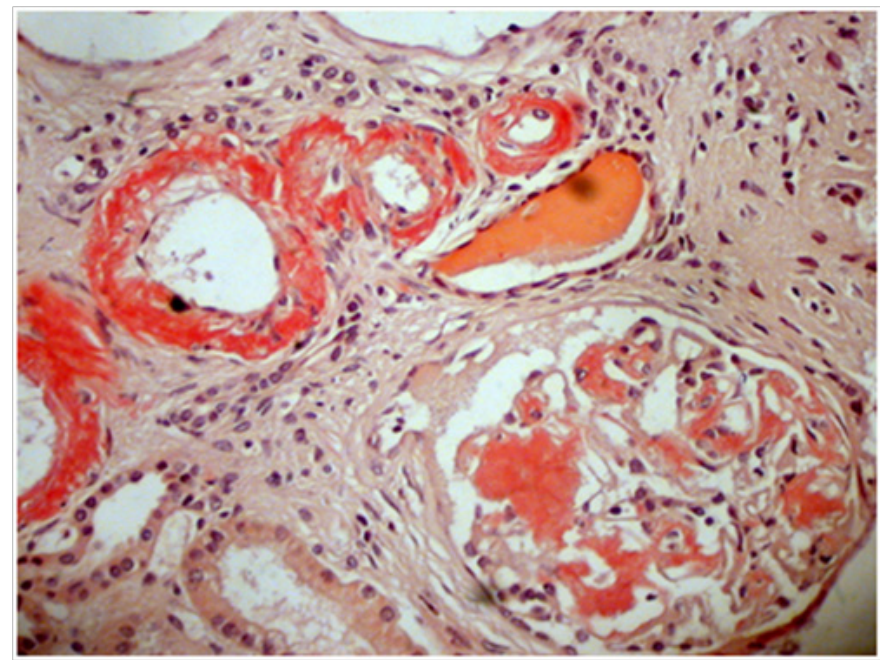

Figure $5 \mathrm{AL}$ amyloidisis, Congo red $\times 250$.

\section{Monoclonal gammopathy of renal significance pathology correlations}

MGRS was represented by "primary" AL amyloidosis, "primary" LCDD/HCDD and also with a variety of other lesions, such as Cryo GN with monoclonal immunoglobulin deposits, PGNMID, MN, CGN, FSGS, TIN and DNS.

Diffuse Nephrosclerosis; FD, franklin's disease; FSGS, focal segmental glomerulosclerosis; GN, glomerulonephritis; HCDD, heavy chain deposition disease; HD, hodgkin's disease; IgM, IMMUNOGLOBULIN M; LCDD, light chain deposition disease; MGRS, monoclonal gammopathy of renal significance; MCD, minimal change disease; MN, membranous nephropathy; NHL/CLL, non-hodgkin lymphoma/leukemia; PGNMID, proliferative GN with monoclonal deposits; SI, specific infiltration; TIN, tubulointerstitial nephritis; WM, waldenström's macroglobulinemia.

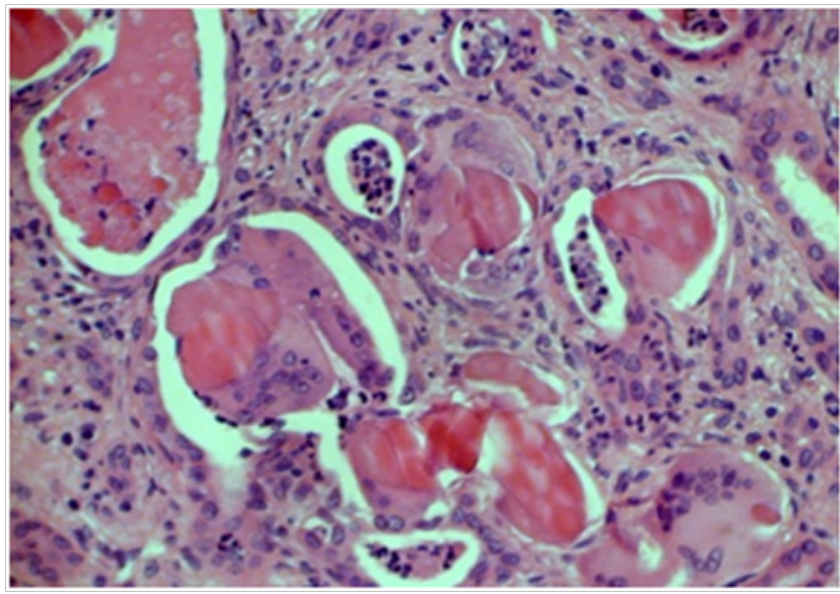

Figure 6 Cast-nephropathy PASx250. 


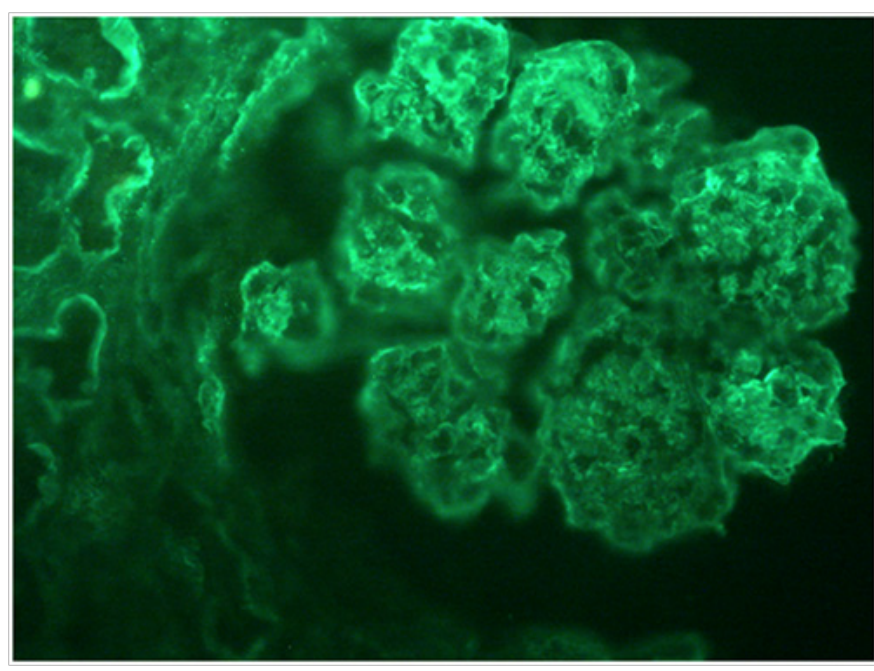

Figure 7 Light chain deposition, immune fluorescence with antibodies against kappa light chains $\times 400$.

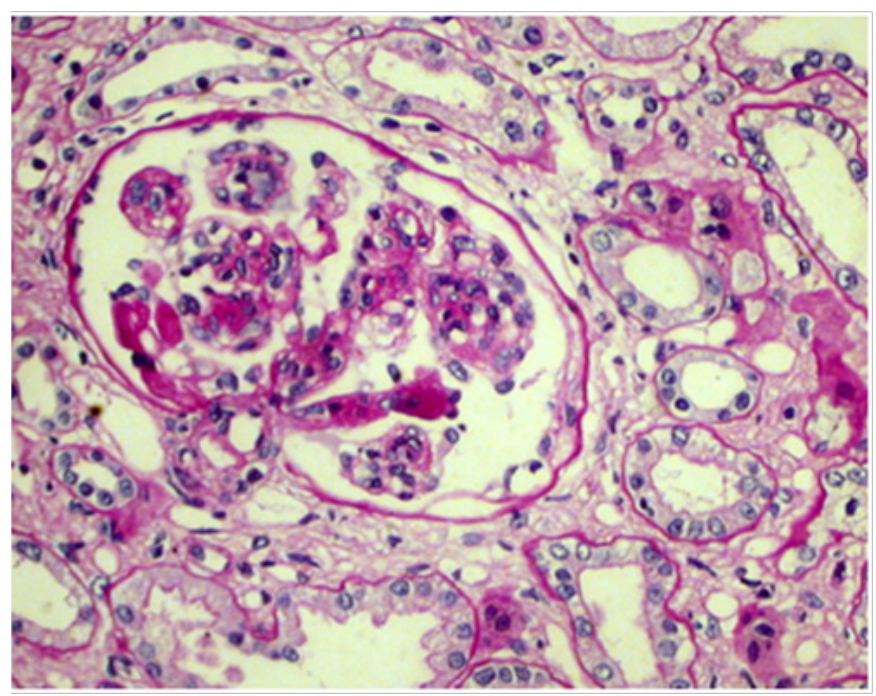

Figure 8 Cryoglobulinemic glomerulonephritis, PASx250.

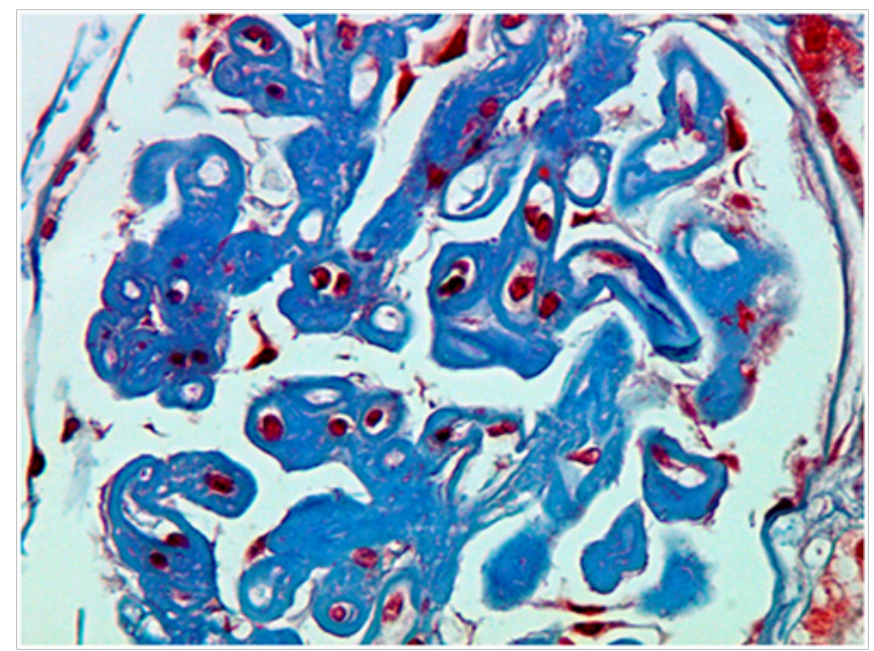

Figure 9 Glomerulonephritis with IgM deposits, Massonx400.

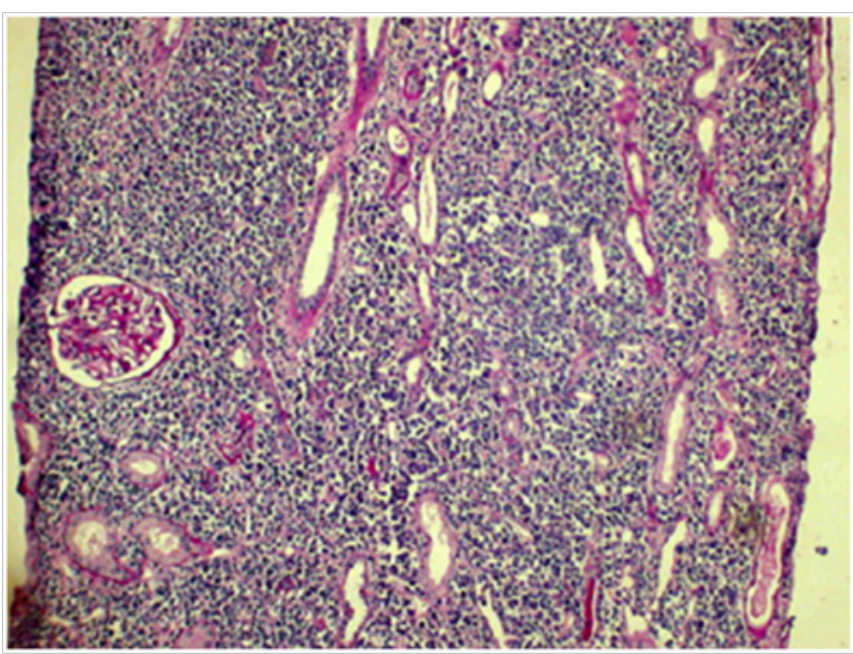

Figure 10 Lymphoid infiltration, PASxI00.

\section{Conclusion}

Our retrospective single-center study of 139 patients with pathology-proven kidney damage confirmed, that patients with lymphoproliferative disorders presenting with renal abnormalities show variety of pathology patterns (mainly, but not exclusively associated with paraprotein deposition), with only few of them predictable on clinical basis. We also confirmed that patients with lowgrade paraprotein secretion demonstrate numerous types of kidney damage beyond the most recognized "primary" amyloidosis, which fully matches with the concept of MGRS. And finally, we confirmed that LPD and MGRS share many clinical and pathology features, reflecting common pathogenesis of renal damage in these conditions. Kidney biopsy, precluded or followed by scrutinous hematological work-up is crucial diagnostic tool for the management of LPD/MGRS patients.

\section{Acknowledgements}

Maria Ratner, Alexander Lokshin, Alexander Vorobjev, Alexey Sukhanov, Mikhail Brodsky, Elena Ipatjeva Arthur Cohen, Olga Vorobjeva, Natalia Tomilina, Aida Melikyan, Elena Tareeva, Olga Vinogradova, Anastasia Tareeva, Tatiana Makarova, Vladimir Lunin, Nuriette Khuajeva, Alexander Kostin, Anna Shubina, Larisa Mendeleeva, AllaKovrigina, Irina Kaplanskaya and Sergey Loginov.

\section{Conflict of interest}

The author declares no conflict of interest.

\section{References}

1. Sanders PW, Booker BB. Pathobiology of cast nephropathy from human Bence Jones proteins. J Clin Invest. 1992;89(2):630-639.

2. Cohen AH. The kidney in plasma cell dyscrasias: Bence-Jones cast nephropathy and light chain deposition disease. Am J Kidney Dis. 1998;32(3):529-532.

3. Korbet SM, Schwartz MM. Multiple myeloma. J Am Soc Nephrol. 2006;17(9):2533-2545.

4. Cambier JF, Ronco P. Onco-nephrology: glomerular diseases with cancer. Clin J Am Soc Nephrol. 2012;7(10):1701-1712. 
5. Da' as N, Polliack A, Cohen Y, et al. Kidney involvement and renal manifestations in non-Hodgkin's lymphoma and lymphocytic leukemia: a retrospective study in 700 patients. Eur J Haematol. 2001;67(3):158-164.

6. Ronco PM. Paraneoplastic glomerulopathies: new insights into an old entity. Kidney Int. 1999;56(1):355-377.

7. Hagler KT, Lynch JW. Paraneoplastic manifestations of lymphoma. Clin Lymphoma. 2004;5(1):29-36.

8. Heher EC, Goes NB, Spitzer TR, et al. Kidney disease associated with plasma cell dyscrasias. Blood. 2010;116(9):1397-1404.

9. Herrera GA, Joseph L, Gu X, et al. Renal pathological spectrum in an autopsy series of patients with plasma cell dyscrasia. Arch Pathol Lab Med. 2004;128(8):875-879.

10. Ronco P, Plaisier E, Aucouturier P. Ig-related renal disease in lymphoplasmacytic disorders: an update. Semin Nephrol. 2009;30(6):557-569.

11. Keeling J, Teng J, Herrera GA. AL-amyloidosis and light-chain deposition disease light chains induce divergent phenotypic transformations of human mesangial cells. Lab Invest. 2004;84(10):1322-1338.
12. Nasr SH, Markowitz GS, Stokes MB, et al. Proliferative glomerulonephritis with monoclonal IgG deposits; a distinct entity mimicking immune-complex glomerulonephritis. Kidney Int. 2004;65(1):85-96.

13. Leung N, Bridoux F, Hutchinson CA, et al. Monoclonal gammopathy of renal significance: when MGUS is no longer undetermined or insignificant. Blood. 2012;120(22):4292-4295.

14. Leung N, Dispenzieri A, Fervenza FC, et al. Renal response after highdose melphalan and stem cell transplantation is a favorable marker in patients with primary systemic amyloidosis. Am J Kidney Dis. 2005;46(2):270-277.

15. Royer B, Arnulf B, Martinez F, et al. High dose chemotherapy in light chain or light and heavy chain deposition disease. Kidney Int. 2004;65(2):642-648.

16. Weichman K, Dember LM, Prokaeva T, et al. Clinical and molecular characteristics of patients with non-amyloid light chain deposition disorders, and outcome following treatment with high dose melphalan and autologous stem cell transplantation. Bone Marrow Transplant. 2006;38(5):339-346. 